\title{
1 Have coral snake mimics diversified more than non-mimics?
}

2

3 Running title: Evolution of coral snake color patterns

4

5 Figures in main text: Figures 1 to 4

6 Figures in Supporting information: Figures S1 to S3

7

8 Data archival location:

9 BEAST XML file and BiSSE MCMC results: $\underline{\text { http://dx.doi.org/10.6084/m9.figshare.831493 }}$

10 R code for analyses and simulations: https://github.com/Caetanods/Dipsadidae color_evolution

12 Authors:

13 Daniel S. Caetano ${ }^{1 \dagger}$, Laura R.V. Alencar ${ }^{2}$, Paulo Passos $^{3}$, Felipe G. Grazziotin ${ }^{4}$, Hussam Zaher ${ }^{4}$, 14 Marcio Martins ${ }^{2}$

15

$16{ }^{1}$ Department of Bioinformatics and Computational Biology, University of Idaho, Life Sciences South

17 252, Moscow, ID, 83843, USA. E-mail: caetanods1@gmail.com

$18{ }^{2}$ Departamento de Ecologia, Universidade de São Paulo, São Paulo, SP, 05508-900, Brazil

$19{ }^{3}$ Departamento de Vertebrados, Museu Nacional, Universidade Federal do Rio de Janeiro, Rio de

20 Janeiro, RJ, 20940-040, Brazil

$21{ }^{4}$ Museu de Zoologia, Universidade de São Paulo, São Paulo, SP, 04263-000, Brazil

$22^{\dagger}$ Corresponding author. 


\section{Abstract}

28 Dipsadidae is the most diversified family of snakes, composed of species showing an impressive

29 variety of color patterns. Some species are cryptic whereas others have contrasting patterns comprised

30 by bright colors alternated with darker shades, including particular combinations of vivid colors

31 characteristic of coral snakes (Elapidae). Species with such patterns are thought to be mimics of coral

32 snakes based on their color pattern similarity, predator avoidance of such patterns in field experiments,

33 and the geographical concordance between models and mimics. Here we test whether color patterns

34 associated with coral snake mimicry and contrasting color patterns in general influenced the

35 diversification dynamics of the group. We compile the largest database of color patterns among reptiles

36 to date, with color descriptions for the majority (594 species) of dipsadids. We used trait-dependent

37 diversification models along with extensive simulations to deal with the recently described statistical

38 bias associated with such methods. Despite the apparent survival advantage associated with coral snake

39 mimicry, we show that there is no detectable influence of color types in the dynamics of diversification

40 in Dipsadidae. We discuss insights into the function of color patterns and argue that non-mimic

41 contrasting patterns might serve as pre-adaptations to mimicry of coral snakes.

42

43 Key words: mimicry, coloration, database, diversification rates, crypsis. 

environment make prey difficult for the predators to detect and recognize (Merilaita and Lind 2005,

46 Stevens and Merilaita 2009). On the other hand, bright and contrasting colors displayed by unpalatable,

47 toxic or venomous animals (i. e., aposematic patterns) serve as warning signals that are often avoided

48 by visually oriented predators (Wallace 1867, Mappes et al. 2005, Speed and Ruxton 2005). However,

49 such conspicuous colors can also be displayed by mimics, which gain protection by deceiving predators

50 that avoid their false warning signals. Strong evidence from field experiments shows that mimicry of

51 warning signals decreases predation pressure when compared to cryptic color patterns (Jeffords et al.

52 1979, Brodie 1993, Brodie and Janzen 1995, Pfennig et al. 2001, Pinheiro 2011, Pfennig et al. 2015).

53 Such reduction in predation pressure may also have positive impacts on habitat use by aposematic

54 lineages and their mimics. Cryptic animals are to some degree restricted to backgrounds which their

55 color patterns match and may only be active at certain times because movement is often antithetic to

56 good crypsis (Speed et al. 2010, Stevens and Ruxton 2012). In contrast, such restrictions may be

57 weaker in aposematic or mimic lineages, which could promote more opportunities to exploit habitat

58 resources (Speed et al. 2010).

59 In contrast with aposematism, the survival advantage of Batesian mimicry is dependent on the

60 relationship between the model and the mimetic organism because predators need to associate the

61 unpalatability or hazard of the model with the warning signals of the deceiver. Once this association is

62 broken, a mimicry breakdown occurs and the mimic phenotype might become maladaptive since

63 warning signals can make individuals more conspicuous to predators (Mallet and Joron 1999, Pfennig

64 et al. 2001, Pfennig et al. 2015). Mimicry breakdown can be caused by allopatry between mimic and

65 model populations as a result of population expansion of the mimic or local extinction of the model

66 (Pfennig and Mullen, 2010). Allopatric mimics are conspicuous to naïve predators that might not avoid

67 their deceptive warning signals and this may result in higher predation rates and eventual extinction of 
68 the mimic population (Pfennig et al. 2015). On the other hand, population expansion or migration of

69 mimics can create opportunities for local adaptation to novel aposematic models. This process could

70 result in selection against intermediate hybrids followed by decreased gene flow among populations

71 and eventually promote reproductive isolation (Mallet and Joron 1999, Pfennig et al. 2015). Over

72 longer time scales such processes might have a positive effect on rates of diversification of mimetic

73 lineages. Previous studies show that aposematic lineages are more species-rich than cryptic ones

74 (Santos et al. 2003, Przeczek et al. 2008), suggesting that the evolution of the aposematic condition

75 may even represent a key innovation (Speed et al., 2010). This key innovation hypothesis could be

76 extended to mimicry; however, the potential effects of mimicry evolution on lineage diversification

77 have yet to be investigated.

78 Among snakes, groups of relatively harmless or mildly venomous species showing color

79 patterns similar to those of venomous coral snakes (Elapidae) have instigated a long debate on whether

80 such patterns are mimetic (see a comprehensive review in Pough 1988). Savage and Slowinski (1992)

81 compiled a remarkable list of coloration descriptions and designed a system of categories to facilitate

82 recognition of coral snake mimics and association with their supposed models. Early reports also relied

83 primarily on the similarity of color patterns between mimics and models to argue in favor of mimicry

84 relationships (Dunn 1954, Hecht and Marien 1956, also see Greene and McDiarmid 1981). Additional

85 evidence came from parallel geographic variation of coral snakes and their putative mimics (e. g.,

86 Hecht and Marien 1956, Zweifel 1960, Greene and McDiarmid 1981, Marques and Puorto 1991) and

87 from field studies using replicas of coral snakes and other similar color patterns (Smith 1975, Brodie

88 1993, Brodie and Janzen 1995, Hinman et al. 1997, Pfennig et al. 2001, Buasso et al. 2006).

89 Some authors pointed to the possibility that contrasting colors, including the stereotypical

90 banded pattern observed in almost all coral snakes, could serve a disruptive function (Gadow 1908,

91 Thayer 1918, Dunn 1954, and Brattstrom 1955). Those reports suggested that the alternate pattern of

92 bands could blend to the background environment and break the outline of the snake body, making 
93 recognition by visually oriented predators difficult. Recently, Titcomb and colleagues (2014) showed

94 that the contrasting ringed pattern of coral snake mimics can create an illusory effect when the

95 individuals are moving fast. The effect, called flicker-fusion, can give advantage to snakes against

96 avian predators independent of mimicry. Despite its protective effect, the plausible disruptive function

97 of the contrasting bands do not invalidate the existence of a mimicry complex between elapids and

98 snakes from other families, since the same color pattern can perform both functions (Titcomb et al.,

99 2014).

101 among snakes, with ca. 700 species occurring from Central to South America (Grazziotin et al. 2012,

102 Uetz and Hosek 2014), and is characterized by an impressive variety of color patterns (see Martins and

103 Oliveira 1998 for some examples). Some dipsadids have color patterns similar to those of coral snakes,

104 and have long been suggested as cases of mimicry of New World coral snakes of the genus Micrurus

105 and Leptomicrurus (family Elapidae; Wallace 1867, Greene and McDiarmid 1981, Sazima and Abe

106 1991, Savage and Slowinski 1992, Martins and Oliveira 1993, Pough 1988, Almeida et al. 2014). The

107 contrasting coloration found in dipsadid snakes always includes bright colors but is not restricted to

108 ringed patterns. In general, species can vary from the coral snake pattern of black, red and yellow rings

109 or bands to a less colorful homogeneous red body with a single black or cream band on the neck

110 (nuchal collar). Besides contrasting color patterns, the family also shows a diverse array of cryptic

111 color patterns, characterized by blotches and shades of brown, gray, or green. Included in the latter are

112 species whose dorsum is cryptic and whose venter has a plain bright color and even a coral snake

113 pattern. Mimetic and cryptic patterns can be found both within and among genera and make dipsadid

114 snakes an ideal study system to investigate the possible effects of such distinct color types on

115 macroevolutionary patterns. 
118 patterns in general) show diverging macroevolutionary patterns when compared to non-mimic and

119 cryptic lineages, respectively. We compile and make available a database with color pattern

120 descriptions for the vast majority of species in the group. We show that there is no detectable influence

121 of supposedly mimic or contrasting color patterns in the dynamics of diversification and argue that

122 non-mimic contrasting color patterns may be pre-adaptations to mimicry of coral snakes.

124 Methods

125 Phylogenetic reconstruction

126 We used sequence data for Dipsadidae and outgroup species available in GenBank (Benson et 127 al. 2014) and previously analyzed by Grazziotin and colleagues (2012, see accession numbers in their

128 Appendix S1). We aligned sequences using MAFFT (Katoh et al. 2005) under the G-INS-i strategy and 129 selected models of molecular evolution for each of the eight gene sequences using a decision theory

130 framework in DT-ModSel (Minin et al. 2003). We concatenated the alignments and set four partitions;

131 one partition for each nuclear gene (bdnf, c-mos, and rag2) and a single partition with the

132 mitochondrial genes (12S, 16S, cytb, nd2, and nd4). We used phyutility (Smith and Dunn 2008) to trim

133 down all sites with $75 \%$ or more missing data and inferred a Maximum Likelihood (ML) tree using

134 GARLI 2.0 (Zwickl 2011). We used the resulting ML phylogeny as the starting tree for three

135 independent searches in BEAST 1.8 (Drummond et al. 2012) for 270 million generations with a

136 thinning interval of 1500 generations each. Since there are sequences available for only few species of

137 each genera we set an incomplete sampling birth-death tree prior (Stadler 2009) and an uncorrelated

138 relaxed clock model to estimate relative branching times. We checked each run for convergence using

139 Tracer 1.6 (Drummond et al. 2012) and excluded 50\% of the posterior chain as burnin. We then

140 combined the posterior set of trees from the three BEAST searches and randomly sampled 100 trees to

141 account for phylogenetic uncertainty in all subsequent analyses. The 100 sampled trees and the BEAST

$142 \mathrm{xml}$ file comprising the data matrix, selected models of molecular evolution, starting tree and prior 
143 parameters is available in FigShare (http://dx.doi.org/10.6084/m9.figshare.831493). We also deposited

144 the configuration and $\log$ files for GARLI 2.0. Figure S1 shows the resulting maximum clade

145 credibility $(\mathrm{MCC})$ tree and respective posterior probability support values.

146

147 Color patterns

148 To understand the evolution of colors and its effect on diversification we compiled the most

149 complete database of coloration patterns for dipsadid snakes. We searched several information sources

150 such as comprehensive taxonomic reviews (e.g., Downs 1967), published articles and books containing

151 photographs of identified individuals (e.g., Savage 2002), trusted on-line photo repositories (e.g.,

152 CalPhotos - http://calphotos.berkeley.edu/ and Reptile Database - http://www.reptile-database.org/),

153 photographs of live specimens, and examination of individuals preserved in scientific collections (e.g.,

154 type specimens). We excluded invalid taxa or names presenting nomenclatural problems that are still

155 appearing in the literature or online databases. We avoided subspecific ranks for coding the currently

156 recognized taxa (with the exception of four subspecies of Alsophis antillensis) because terminals in

157 available phylogenies correspond to species only and less than $10 \%$ of the members of the family

158 Dipsadidae present valid subspecies to date.

159 While color diversity makes the family Dipsadidae interesting for studies focusing on the

160 evolution of color patterns such as ours, this is also the most challenging characteristic of the system.

161 Since it is not possible to consider all diversity of color patterns for comparative analyses, we used

162 broad categories that are directly related to the hypotheses tested. In a first test, we used the categories

163 coral-mimic and non-mimic (see Figure 1 and database available in

164 http://dx.doi.org/10.6084/m9.figshare.831493). We call coral-mimics species that resemble the pattern

165 of any New World coral snake species (see Roze 1996, Campbell and Lamar 2004). Species included in

166 this category can show the coral-mimic pattern throughout the dorsum (e.g., Simophis rhinostoma) or

167 restricted to the anterior portion of the body (e.g., Pseudoboa coronata). It is impossible to elect 
species-specific mimicry hypotheses for all 121 species included in the coral-mimic category given our

169 current knowledge of the ecology and geographical distribution of the group. Therefore, we assigned

170 species based on their color pattern similarity with putative models. On the other hand, all species not

171 defined as potential mimics of coral snakes, independent of whether their color pattern was better

172 described as contrasting or cryptic, were included in the category of non-mimics. As a result, the non-

173 mimic category comprise species with cryptic color patterns and others with bright coloration but not

174 resembling any known lineage of New World coral snake.

176 such as hues and blotches of brown or green and bright colored patterns, which are likely to be more

177 conspicuous to a visually oriented predator. One might argue that such definition is too inclusive, and

178 the same category comprises different defensive strategies. In this light, we performed separate

179 analyses using species that show brightly colored patterns in general, independent of whether the color

180 pattern was similar to those of coral snakes. We defined such lineages as contrasting. Given our

181 definitions, the category coral-mimic is a subset of the contrasting category; every coral-mimic lineage

182 is among the species defined as contrasting, but the reverse is not true. We included species not

183 classified as contrasting into a single category comprising cryptic patterns. Cryptic coloration has been

184 defined as any pattern resembling a random sample of the habitat background (Endler 1986). However,

185 since we do not have accurate habitat descriptions for most of species, we defined as cryptic all color

186 patterns lacking contrasting colors (with exception of arboreal snakes, see below). Examples of such

187 patterns are blotches with hues of brown, reddish brown, gray, and other combinations of dark colors.

188 We also considered as cryptic species whose dorsum is homogeneously green, since individuals of

189 those species are usually found among leaves of trees and bushes (e.g., Uromacer). Some Pseudoboini

190 snakes (sensu Zaher et al. 2009) show ontogenetic changes in color pattern in which juveniles are

191 brightly colored but become cryptic when adults (Martins and Oliveira 1998). We included those

192 species into the contrasting category since juveniles correspond to the life stage most threatened by 
predation (Bonnet et al. 1999) and thus their defensive tactics are fundamental for individuals to reach

194 sexual maturity. Some species show cryptic color patterns in the dorsum but have contrasting patterns

195 restricted to the venter. The distinct patterns in the dorsum and venter are usually associated with a

196 threatening display in which individuals twist the body and expose the bright colors when disturbed

197 (Martins and Oliveira 1993, Sawaya et al. 2008, Tozetti et al. 2009). Hence, we classified those cases

198 into the contrasting category instead of following their dorsal coloration. We performed additional

199 analyses in which those species were classified as cryptic, but found no appreciable difference in

200 results.

Some species are known to show color polymorphism. In such cases populations may show

202 cryptic patterns occasionally associated to thermoregulation (Tanaka 2005, but see Lorioux et al. 2008).

203 Alternatively, contrasting colors in polymorphic populations can be due to increasing sexual

204 dichromatism in the course of the reproductive season (Forsman 1995, Lindell and Forsman 1996),

205 related to non-selective processes, such as migration and dispersal (King and Lawson, 1995) or genetic

206 drift in local (Brakefield, 1990) or island populations (Bittner and King, 2003). Independent of the

207 potential sources of the polymorphic color patterns we assigned species to the cryptic category every

208 time a cryptic morph was described among the color types.

210 Comparative analyses

211 We sampled 100 trees from the posterior distribution of the BEAST analysis, rescaled all trees

212 to a total depth of 1 , and retained only one randomly selected species of each genus while pruning the

213 rest. When the original tree had paraphyletic genera we selected the most inclusive monophyletic clade

214 representing each group and kept a single species to represent each of those genera. We used the

215 terminally unresolved trees (FitzJohn et al. 2009), the number of species of each genus and their color

216 patterns to test hypotheses of trait-dependent diversification using the Binary State Speciation and

217 Extinction model (BiSSE - Maddison et al. 2007, FitzJohn et al. 2009, FitzJohn 2012). We repeated all 
218 BiSSE analyses based on the two color categorizations: coral-mimic versus non-mimic and contrasting

219 versus cryptic. We used Markov chain Monte Carlo (MCMC) to estimate the posterior distribution of

220 the parameters for the unconstrained BiSSE model (six free parameters), in which speciation $(\boldsymbol{\lambda})$ and

221 extinction $(\boldsymbol{\mu})$ are estimated dependent on the color pattern of the lineages, and the constrained model,

222 in which $\lambda$ and $\boldsymbol{\mu}$ are not related to color types (four free parameters). We used an exponential prior

223 distribution with rate parameter equal to 0.3 for all BiSSE parameters and a starting point equal to the

224 maximum likelihood estimate (MLE). We ran 10,000 generations of MCMC (chain length was based

225 on preliminary analyses), discarded $50 \%$ of generations as burnin and checked convergence using the

226 'coda' package (Plummer et al. 2006). To test whether the full model explained the data better than the

227 trait-independent model we performed model selection using the Bayesian Deviance Information

228 Criteria (DIC - Gelman et al. 2014). We performed all analyses with both the coral-mimic versus non-

229 mimic and the contrasting versus cryptic categories.

230 In addition to the BiSSE model, which estimates homogeneous diversification rates across the

231 tree, we used Medusa (Alfaro et al. 2009) to test whether rates of diversification change independent of

232 color types. This model estimates rates of diversification and jump locations in a given phylogeny

233 using a stepwise AIC approach to sequentially fit models of diversification with increased number of

234 jumps in rates of speciation and extinction. In order to incorporate the effect of uncertainty in topology

235 and branch lengths we applied Medusa across all 100 sampled trees. However, there is no simple

236 solution to summarize those results, since distinct tree topologies have unique sets of nodes. Thus, we

237 chose to focus only in the rate estimates for the external branches (i.e., the genera). This approach

238 allows us to compare groups of genera showing consistently higher or lower rates of diversification

239 with respect to the background rate, ignoring information about the specific position of each jump.

241 significant results even when there is no true relationship between traits and shifts in diversification

242 rates. We performed simulations to correct our results for this deviation. Since the BiSSE model 
influences the dynamics of diversification and makes it difficult to simulate trait data under a fixed tree,

244 each simulation generated both trait data and phylogeny. First, we did posterior predictive checks for

245 model adequacy to test whether data simulated under the trait-dependent and trait-independent BiSSE

246 models are similar to the observed data. For each model, we simulated traits using the 'tree.bisse'

247 function in the package 'diversitree' (FitzJohn 2012) with parameters drawn from the joint posterior

248 distribution resulting from the BiSSE analysis and constraining simulations to have a tree depth equal

249 to 1 (identical to the empirical trees). Finally, we compared the number of species and the relative

250 frequencies of each trait generated by the simulations with the empirical dataset. If models are

251 adequate, simulated phylogenies should produce both diversity and frequency of states similar to the

252 observed data.

254 new datasets by drawing from a binomial distribution with probabilities equal to the frequency of each

255 trait in the observed data. We sampled 10 trees from the posterior distribution that resulted from the

256 BEAST analysis and performed an MLE of the BiSSE model (trait-independent and full model) using

257 each of the simulated datasets. We computed the likelihood ratio test for each BiSSE estimate and

258 produced a distribution of $p$ values for each of the 10 sampled trees. This distribution represents the

259 expected $\mathrm{p}$ values for the likelihood ratio test when traits have no effect in diversification. Following,

260 we tested whether $\mathrm{p}$ values obtained in the model test using the observed data and the same trees are

261 significantly smaller than the distribution under the null model (i.e., data generated from a binomial

262 distribution).

To perform ancestral state estimates, we calculated the grand mean of the posterior

264 distribution of transition rates $\left(\mathbf{q}_{\mathbf{0 1}}\right.$ and $\left.\mathbf{q}_{\mathbf{1 0}}\right)$ across all 100 sampled trees under the accepted model after

265 performing the BiSSE simulations. Then we used those values to infer the MLE of the marginal

266 ancestral states reconstruction on the maximum clade credibility (MCC) tree. We performed all 
267 comparative analyses in R (R Core Team 2015). Scripts to replicate analyses, simulations, and figures

268 are available in https://github.com/Caetanods/Dipsadidae_color_evolution.

270 Results

271 We compiled the largest report of coloration descriptions for reptiles comprising data for 594

272 species of dipsadid snakes and covering over $80 \%$ of the known diversity of the group. We were able to

273 get detailed color descriptions for most species, but for some the information available was incomplete

274 or limited (i.e., taxa known from a single specimen). Although those cases were not suitable for

275 definition as coral-mimic or non-mimic lineages (i.e., state unknown or 'NA'), we managed to classify

276 those as either contrasting or cryptic for all but a few exceptions. Among all data sources, museum

277 specimens are the most difficult to categorize since colors fade after preservation and only light and

278 dark hues remain. Bright colors such as yellow, orange, pink, and red (derived from carotenoid

279 pigments) fade completely over time turning into cream on preservative fluid. In contrast, dark

280 pigmentation is preserved and sometimes turns into shades of black or dark brown. As a result, we

281 assigned museum specimens with alternate bright and dark bands (or with a distinct nuchal collar) as

282 contrasting and homogeneous light or dark patterns as cryptic. We provide the species list and their

283 color patterns under both categorization schemes in the online data repository

284 (https://github.com/Caetanods/Dipsadidae_color_evolution).

We used the BiSSE model to investigate whether color patterns have an effect on the

286 diversification rates of the snake family Dipsadidae. We made analyses based on two different

287 categorizations of the same dataset: coral-mimics versus non-mimics, which tested for differences in

288 macroevolutionary patterns between lineages with and without color patterns similar to New World

289 coral snakes, and contrasting versus cryptic color patterns, which investigated changes in

290 diversification associated with the presence or absence of bright coloration. When we compared coral-

291 mimic to non-mimic patterns, the BiSSE analysis showed that coral-mimic lineages have net 
292 diversification rates $\left(\boldsymbol{\lambda}_{\mathbf{1}}-\boldsymbol{\mu}_{1}\right)$ in average two times higher than non-mimics (Figure 2$)$. When

293 diversification rates are constrained to be independent of color types we recovered intermediate net

294 diversification values relative to the trait-dependent model. These results are similar to the analyses

295 based on contrasting versus cryptic color patterns (Figure 2). Both the trait-independent and trait-

296 dependent diversification models estimated strongly asymmetrical transition rates with changes from

297 the coral-mimic to the non-mimic state $\left(\mathbf{q}_{10}\right)$ in average two to three times more frequent than the

298 reverse $\left(\mathbf{q}_{01}\right)$. Median transition rates are qualitatively comparable independent of color categorization

299 (Figure 3). On the other hand, the posterior distributions of transition rates for coral-mimics versus

300 non-mimics are wider than contrasting versus cryptic, showing more uncertainty in parameter

301 estimates. Results were consistent across all sampled trees and in all cases the state-dependent

302 diversification model was the one preferred by the DIC model selection criteria (Figure S2).

303 Results from the Medusa analyses, which estimate shifts in diversification rates independent

304 of color types, indicate that the preferred model across all 100 trees was the Yule diversification model

305 (Yule 1924), which has a single birth parameter in the absence of extinction. The estimated median rate

306 is comparable across almost all genera, but some clades have a tendency for higher rates of

307 diversification when compared to background rates (Figure S3). While most of the 100 sampled trees

308 were estimated to have homogeneous rates of diversification, a portion of these trees shows evidence

309 for higher rates that are consistently shared by a set of genera. Ten genera of the tribe Alsophiini, five

310 genera of Tachymenini, and a clade formed by Pseudoeryx and Hydrops show rate distributions with a

311 tendency for higher rates of diversification, although median values are not significantly distinct from

312 background rates (Figure S3).

314 ratio tests (LRTs) to evaluate whether the BiSSE trait-dependent diversification model is adequate to

315 explain the observed data and if preferring the more complex model is justifiable. Since there is no

316 appreciable difference in parameter estimates and DIC results across the categorizations of color types, 
317 we performed simulations based only in the contrasting and cryptic categories. The posterior predictive

318 simulations using both BiSSE models (trait-dependent and trait-independent) produced trees much

319 smaller than the 594 species of the empirical tree (see left column of Figure 4). Since the stopping

320 criteria for simulations were tree depth, the number of species in each simulated tree was free to vary.

321 Trees simulated under the full model had on average 211 species and only $9 \%$ of those showed more

322 species than the observed data. Similarly, trees simulated using the trait-independent model had on

323 average 186 species, of which only 5\% were larger than the observed data. With respect to trait

324 frequency, both models simulated datasets biased towards higher frequencies of the cryptic color type.

325 On average the full model had $67 \%$ and the trait-independent model $84 \%$ of the simulations showing

326 frequencies of the cryptic color type higher than observed in the empirical data (see right column of

327 Figure 4). Therefore, our results show that both models have similar biases and the characteristics of

328 the data not satisfactorily explained by the BiSSE model are independent to whether rates of

329 diversification are associated with color types.

Although parameter estimates under the BiSSE model are robust to our different

331 categorization of color patterns, the ML ancestral state estimate is dependent on whether we consider

332 the coral-mimic or contrasting color type categories. Ancestral estimates considering species as coral-

333 mimic or non-mimic suggest the root node had a non-mimic color pattern and just two clades show high

334 likelihood for coral-mimic ancestors (Figure 1). However, when species are categorized as contrasting

335 or cryptic, the root node is reconstructed as most likely contrasting and several transitions to the

336 cryptic form occurred throughout the tree with no evidence of reversals (Figure 1). 


\section{Discussion}

\section{Model selection and (in)adequacy}

We fitted the trait-dependent and trait-independent BiSSE models to the data and performed

342 model selection using the Deviance Information Criteria (DIC). DIC showed strong support for the

343 trait-dependent model for both color pattern categorizations (coral-mimic versus non-mimic and

344 contrasting versus cryptic). However, our simulations showed that there is no evidence to choose the

345 trait-dependent model over the trait-independent model. Likelihood ratio tests on simulated data

346 consistently gave support to the trait-dependent diversification model (with threshold value of 0.05 )

347 despite the fact that data were generated using draws from a binomial distribution, independent of the

348 phylogeny. The BiSSE method is likely to support the trait-dependent model even when there is no real

349 effect of traits on rates of diversification, supporting findings by Rabosky and Goldberg (2015). The

350 choice of the full model by the DIC approach was unjustifiable and might be a statistical artifact.

351 Hence, our preferred model is the trait-independent model in which color type has no influence on

352 diversification.

354 BiSSE models share similar biases. Both BiSSE models are equally inadequate in explaining the

355 observed data whereas the LRT simulations provide evidence that the best model is the simpler model

356 rather than the model in which rates of diversification depend on color type. Results from the Medusa

357 analyses also corroborate this conclusion. There is no support for shifts in rates of diversification and

358 the majority of lineages showing a tendency for higher rates are comprised of cryptic species (Figure

359 S3). At the moment, unlike other models of trait evolution (Pennell et al. 2014), it is not clear which set

360 of summary statistics can be used to assess the adequacy of BiSSE models. More studies are needed to

361 better understand the scenarios under which this and other models of the xxSSE family are prone to

362 misbehave and elect a set of informative summary statistics for predictive posterior checks. 


\section{Uncertainty in ancestral state estimates}

365 for the BiSSE model under both color categorizations (Figures 2 and 3) is most likely an effect of the

366 proportion of states in each of the genera for each categorization. Given our color category definitions,

367 every coral-mimic was also included in the contrasting category, but the contrary is not true. The

368 proportion of contrasting species is higher than coral-mimics in several genera and may have increased

369 the likelihood of nodes estimated as contrasting when comparing to the same nodes under the coral-

370 mimic and non-mimic categorization. In both cases changes in color pattern are mostly concentrated

371 within genera, with exception of the tribes Alsophiini and Tachymenini, both comprising only cryptic

372 (non-mimic) species (marked respectively in green and red in Figure 1). In those tribes the transition

373 from contrasting to cryptic forms may have occurred at the base of the clades with reversals restricted

374 to the genus Ialtris (tribe Alsophiini). These results need to be interpreted with caution, since ancestral

375 estimates are often misleading and a fully resolved tree for the group might falsify our reconstructions

376 by showing reversals from coral-mimic/contrasting to non-mimic/cryptic color types present at the

377 genus level.

378

379 Color patterns have no effect on the diversification of dipsadid snakes

381 been intensely debated since the 50s (Dunn 1954, Hecht and Marien 1956). However, only a few

382 studies have investigated the evolution of snake color patterns using an explicit phylogenetic approach

383 (e.g., Pyron and Burbrink 2009) despite recent advances in comparative methods. Our results show no

384 appreciable effect of the color types in diversification rates despite the impressive diversity of color

385 patterns found in the group. Estimates of diversification rates independent of color types also give

386 support to the trait-independent model, since results do not suggest any concurrence of shifts in

387 diversification rates with the presence of coral-mimic or contrasting patterns. Overall, our results 
suggest that the ecological functions of coral-mimic and non-mimic color types in dipsadid snakes have

389

390

391

392

393

394

395

396

397

398

399

400

401

402

403

404

405

406

407

408

409

410

411 mimetic and disruptive coloration (Dunn 1954, Titcomb et al. 2014). The disruptive function (or

412 flicker-fusion effect) can prevent or mitigate maladaptation caused by allopatric distribution of mimics 
413 and models, since it provides protection even in the presence of naïve predators. The double function of

414 the contrasting pattern as mimetic and illusionary may help explain the impressive diversity of colors

415 and patterns found among dipsadid snakes. One plausible explanation is that contrasting patterns may

416 serve as pre-adaptations to the evolution of coral snake mimicry in certain lineages.

417 Ancestral node reconstructions of the contrasting pattern in dipsadids show that most genera

418 are likely to have some form of contrasting colored ancestor (Figure 1). This pattern changes when

419 considering only the subset of contrasting lineages with color patterns similar to coral snakes. In the

420 latter case, reconstructions show that transitions most likely occurred exclusively within genera in 13

421 out of 24 genera comprising coral-mimic lineages. Among the genera estimated to have coral-mimic

422 ancestors 9 out of 11 belong to the Pseudoboini, characterized by a single transition to the coral-mimic

423 type (Figure 1). Since coral-mimics are a subset of contrasting lineages and contrasting ancestors occur

424 in nodes deeper in the tree than the coral-mimics ancestors, it is likely that contrasting patterns not

425 resembling true coral snakes predate the supposed mimetic patterns. The disruptive effect of some

426 contrasting patterns, especially those including light and dark bands, might confer protection against

427 predation and can show rudimentary similarities to potential coral snake models. This scenario could

428 lead to a mimicry relationship as predation pressure can produce a directional selective gradient pulling

429 towards color patterns more similar to those of the model lineage. Although our results suggest this

430 relationship, the current knowledge of the Dipsadidae phylogeny does not provide enough resolution to

431 test this hypothesis, since changes from contrasting to cryptic patterns occurred almost exclusively

432 within genera.

433

434 Ecological opportunity on the West Indies

435 We estimated rates of diversification using a model that is agnostic to color types (Medusa)

436 and two clades showed a tendency for elevated rates of diversification, the tribe Alsophiini and part of

437 the tribe Tachymenini (Figure S3). Both tribes comprise cryptic species, with exception of the genus 
Ialtris. The Alsophiini is an interesting case. It most likely dispersed from mainland South America

over the ocean to the West Indies, where it is endemic (Maglio 1970, Hedges et al. 2009). The West

440 Indies are known by the remarkable adaptive radiation of Anolis lizards (Losos and Ricklefs 2009) and

441 might also have provided ecological opportunities for the radiation of other squamate reptiles. For

442 instance, Burbrink and colleagues (2012) showed that the diversification of the Alsophiini in the West

443 Indies bears no signal of increased rates of diversification. In contrast, our results show a tendency for

444 increased rates of diversification associated with the same group. Absence of such signal in results by

445 Burbrink and colleagues (2012) may be a reflection of including only the Alsophiini in the analysis

446 whereas herein we used phylogenies including the whole family. The increased rate of diversification

447 of the Alsophiini may be, such as hypothesized by Burbrink et al. (2012), a function of the opportunity

448 for ecological diversification and not associated with the concurrent change from contrasting to cryptic

449 color patterns. It is important to note that the monophyly of the tribe has been contested by some

450 studies (Grazziotin et al. 2012, Pyron et al. 2013) and taxonomic rearrangements are likely to occur in

451 the future. It is possible that tests based on a revised taxonomy and a finer phylogenetic resolution may

452 show support for the hypothesis of ecological opportunity.

453

454 Concluding remarks

Herein we compiled from primary sources and made available a database of color patterns for

456594 species of dipsadid snakes, the largest compilation of color descriptions for reptiles to date. We

457 found that coral-mimic or contrasting patterns have no significant effect on rates of diversification

458 when compared to non-mimic or cryptic color types. This is an intriguing contrast with the fact that

459 aposematic clades are more species-rich than their cryptic sister groups (Przeczek et al. 2008).

460 Speciation or extinction of mimetic lineages are theoretically linked to the relationship with their

461 models. However, this dependence can be loosened if the mimetic trait is associated with a secondary

462 protective function. Both eventual extinction events caused by allopatry with models and speciation as 
463 a result of local adaptation to novel models can be 'buffered' by the secondary function of the trait. The

464 protection by illusion might be a precursor for both the remarkable convergence of snake lineages to

465 coral-like forms and the maintenance of mimicry despite the supposed likelihood of mimicry

466 breakdowns.

467 It is naïve to think that a unique set of traits, such as color patterns, can reflect all relevant

468 factors that drive the dynamics of diversification of any group. A more detailed analysis of our

469 questions could be accomplished by overlapping evolutionary patterns of the Dipsadidae with those of

470 New World coral snakes, for example. However, both phylogenetic data and suitable comparative

471 models are not yet available. Further appreciation of transitions between contrasting and cryptic color

472 patterns within dipsadid genera can shed light on whether disruptive colors can serve as a pre-

473 adaptation to mimicry and help insert new pieces into the coral snake mimicry puzzle. Understanding

474 under which phylogenetic and ecological scenarios mimicry is likely to evolve is a key factor to explain

475 the patterns of phenotypic convergence observed among distantly related lineages across the tree of

476 life.

477

478 Acknowledgments

479 We would like to thank Denim Jochimsen, Josef Uyeda, Matt Pennell, Rafael Maia, and Andrew

480 Kraemer for extensive discussions about snakes and statistics. Special thanks to Luke Harmon, DSC

481 advisor, for insightful comments on the work and manuscript. This project applied many ideas

482 discussed at the Phylogenetics Reading Group (PuRGe) at the University of Idaho. DSC was supported

483 by a fellowship from Coordenação de Aperfeiçoamento de Pessoal de Nível Superior (CAPES:

484 1093/12-6). LRVA was supported by Fundação de Amparo a Pesquisa do Estado de São Paulo

485 (FAPESP, 2012/03038-2). Financial support to PP was provided by Conselho Nacional de

486 Desenvolvimento Científico e Tecnológico (CNPq, 482086/2012-2 and 8256995713198058) and

487 Fundação de Amparo a Pesquisa do Estado do Rio de Janeiro (E-26/110.434/2012 and E- 
488

489

490

491

492

493

494

495

496

497

498

499

500

501

502

503

504

505 Brattstrom, B. H. 1955. The coral snake "mimic" problem and protective coloration. Evolution. 9:217-

506

507 Brodie III, E. D. 1993. Differential avoidance of coral snake banded patterns by free-ranging avian

508

509 Brodie III, E. D., and F. J. Janzen. 1995. Experimental studies of coral snake mimicry: generalized

510

Harmon. 2009. Nine exceptional radiations plus high turnover explain species diversity in jawed vertebrates. Proc Natl Acad Sci USA. 106:13410-13414.

Almeida, P. C., D. T. Feitosa, P. Passos, and A. L. C. Prudente. 2014. Morphological variation and taxonomy of Atractus latifrons (Günther, 1868) (Serpentes: Dipsadidae). Zootaxa. 3860:64-80.

Benson, D. A., K. Clark, I. Karsch-Mizrachi, D. J. Lipman, J. Ostell, and E. W. Sayers. 2014. GenBank. Nucleic Acids Res. 42:D32-37.

Bittner, T. D., and R. B. King. 2003. Gene flow and melanism in garter snakes revisited: a comparison of molecular markers and island. vs. coalescent models. Biol J Linn Soc. 79:389-399.

Bonnet, X., G. Naulleau, and R. Shine. 1999. The dangers of leaving home: dispersal and mortality in snakes. Biol Conserv. 89:39-50.

Brakefield, P. M. 1990. Genetic drift and patterns of diversity among colour-polymorphic populations of the homopteran Philaenus spumarius in a island. archipelago. Biol. J. Linn Soc. 39:219-237. 219. predators in Costa Rica. Evolution. 47:227-235. avoidance of ringed snake patterns by free-ranging avian predators. Funct Ecol. 9(2):186-190. 
511 Buasso, C. M., G. C. Leynaud, and F. B. Cruz. 2006. Predation on snakes of Argentina: Effects of coloration and ring pattern on coral and false coral snakes. Stud Neotrop Fauna Environ. 41:183-

513 188.

514 Burbrink, F. T., S. Ruane, and R. A. Pyron. 2012. When are adaptive radiations replicated in areas?

515 Ecological opportunity and unexceptional diversification in West Indian dipsadine snakes

516 (Colubridae: Alsophiini). J Biogeogr. 39:465-475.

517 Campbell, J.A. and W.W. Lamar. 2004. The Venomous Reptiles of the Western Hemisphere. 2

518 Volumes. Cornell University Press, Ithaca, NY.

519 Downs, F. L. 1967. Intrageneric relationships among colubrid snakes of the genus Geophis Wagler.

$520 \quad$ Misc Publ Mus Zool Univ Mich. 131:1-188.

521 Drummond, A. J., M. A. Suchard, D. Xie, and A. Rambaut. 2012. Bayesian phylogenetics with BEAUti 522 and the BEAST 1.7. Mol Biol Evol. 29(8):1969-1973.

523 Dunn, E. R. 1954. The coral snake "mimic" problem in Panama. Evolution. 8:97-102.

524 Endler, J. A. 1986. Natural selection in the wild. Princeton Univ. Press, Princeton, NJ.

525 FitzJohn, R. G. 2012. Diversitree: Comparative phylogenetic analyses of diversification in R. Methods 526 Ecol Evol. 3:1084-1092.

527 FitzJohn, R. G., W. P. Maddison, and S. P. Otto. 2009. Estimating trait-dependent speciation and 528 extinction rates from incompletely resolved phylogenies. Syst Biol. 58:595-611.

529 Forsman, A. 1995. Opposing fitness consequences of colour pattern in male and female snakes. J Evol $530 \quad$ Biol. 8:53-70.

531 Gadow, H. 1908. Through southern Mexico. Witherby and Co. Press, London, UK.

532 Gelman, A., Hwang J., and Vehtari A. 2014. Understanding predictive information criteria for Bayesian 
534 Grazziotin, F. G., H. Zaher, R. W. Murphy, G. Scrocchi, M. A. Benavides, Y. P. Zhang, and S. L.

535 Bonatto. 2012. Molecular phylogeny of the New World Dipsadidae (Serpentes: Colubroidea): a

536 reappraisal. Cladistics 28:437-459.

537 Greene, H. W., and R. W. McDiarmid. 1981. Coral snake mimicry: does it occur? Science 213:1207-

539 Hecht, M. K., and D. Marien. 1956. The coral snake mimic problem: a reinterpretation. J Morphol 98:335-365.

541 Hedges, S. B., A. Couloux, and N. Vidal. 2009. Molecular phylogeny, classification, and biogeography 542 of West Indian racer snakes of the Tribe Alsophiini (Squamata, Dipsadidae, Xenodontinae). Zootaxa 2067:1-28.

544 Hinman, K. E., H. L. Throop, K. L. Adams, A. J. Dake, K. K. McLauchlan, and M. J. McKone. 1997.

545 Predation by free-ranging birds on partial coral snake mimics: the importance of ring width and $546 \quad$ color. Evolution 51:1011-1014.

547 Jeffords, M. R., J. G. Sternburg, and G. P. Waldbauer. 1979. Batesian mimicry: field demonstration of 548 the survival value of pipevine swallowtail and monarch color patterns. Evolution 33:275-286.

549 Katoh, K., K. Kuma, H. Toh, and T. Miyata. 2005. MAFFT version 5: improvement in accuracy of $550 \quad$ multiple sequence alignment. Nucleic Acids Res. 33:511-518.

551 King, R. B., and R. Lawson. 1995. Color pattern variation in Lake Erie water snakes: the role of gene 552 flow. Evolution 47:1819-1833.

553 Lindell, L. E. and A. Forsman. 1996. Sexual dichromatism in snakes: support to flicker-fusion 554 hypothesis. Can J Zool. 74:2254-2256.

555 Lorioux, S., X. Bonnet, F. Brischoux, and M. De Crignis. 2008. Is melanism adaptive in sea kraits? Amphib-Reptilia. 29:1-5.

557 Losos, J. B., and R. E. Ricklefs. 2009. Adaptation and diversification on islands. Nature 457:830-836. 
558 Maddison, W. P., P. E. Midford, and S. P. Otto. 2007. Estimating a binary character's effect on speciation and extinction. Syst Biol. 56:701-710.

560 Maglio, V. J. 1970. West Indian xenodontine colubrid snakes: their probable origin, phylogeny, and zoogeography. Bull Mus Comp Zool. 141:1-54.

562 Mallet, J., and M. Joron. 1999. Evolution of diversity in warning color and mimicry: polymorphisms, $563 \quad$ shifting balance, and speciation. Annu Rev Ecol Syst. 30:201-233.

564 Mappes, J., N. Marples, and J. A. Endler. 2005. The complex business of survival by aposematism. 565 Trends Ecol Evol. 20(11):598-603.

566 Marques O. A. V., and Puorto, G. 1991. Padrões cromáticos, distribuição e possível mimetismo em 567 Erythrolamprus aesculapii (Serpentes, Colubridae). Mem Inst Butantan. 53(1):127-134.

568 Martins, M., and M. E. Oliveira. 1993. The snakes of the genus Atractus Wagler (Reptilia: Squamata:

569 Colubridae) from the Manaus region, central Amazonia, Brazil Zool Med. 67:21-40.

570 Martins, M., and M. E. Oliveira. 1998. Natural history of snakes in forests of the Manaus region, cen571 tral Amazonia, Brazil. Herp Nat Hist. 6:78-150.

572 Merilaita, S., and J. Lind. 2005. Background-matching and disruptive coloration, and the evolution of 573 cryptic coloration. Proc R Soc Lond B Biol Sci. 272:665-670.

574 Minin, V., Z. Abdo, P. Joyce, and J. Sullivan. 2003. Performance-based selection of likelihood models 575 for phylogeny estimation. Syst Biol. 52:674-683.

576 Pennell, M. W., R. G. FitzJohn, W. K. Cornwell, and L. J. Harmon. 2015. Model adequacy and the 577 macroevolution of angiosperm functional traits. Am Nat. 186(2):E33-E50.

578 Pfennig, D. W., and S. P. Mullen. 2010. Mimics without models: causes and consequences of allopatry 579 in Batesian mimicry complexes. Proc R Soc Lond B Biol Sci. 277:2577-2585.

580 Pfennig, D. W., W. R. Harcombe, and K. S. Pfennig. 2001. Frequency-dependent Batesian mimicry. $581 \quad$ Nature 410:323-323. 
582 Pfennig, D. W., C. K. Akcali, and D. W. Kikuchi. 2015. Batesian mimicry promotes pre- and post583 mating isolation in a snake mimicry complex. Evolution 69:1085-1090.

584 Pinheiro, C. E. G. 2011. On the evolution of warning coloration, Batesian and Müllerian mimicry in 585 Neotropical butterflies: the role of jacamars (Galbulidae) and tyrant-flycatchers (Tyrannidae). J $586 \quad$ Avian Biol. 42:277-281.

587 Plummer M., N. Best, K. Cowles, and K. Vines. 2006. CODA: convergence diagnosis and output $588 \quad$ analysis for MCMC. R News. 6:7-11.

589 Pough, F. H. 1988. Mimicry and related phenomena. In: Biology of Reptilia, vol. 16, Ecology B, 590 Defence and Life History, p. 154-234. Gans, C., Huey, R.B., Eds, New York, Allan R. Liss Inc., $591 \quad$ New York.

592 Przeczek, K., C. Mueller, and S. M. Vamosi. 2008. The evolution of aposematism is accompanied by 593 increased diversification. Integr Zool. 3:149-156.

594 Pyron, R. A., and F. T. Burbrink. 2009. Body size as a primary determinant of ecomorphological 595 diversification and the evolution of mimicry in the lampropeltinine snakes (Serpentes: 596 Colubridae). J Evol Biol. 22:2057-2067.

597 Pyron, R. A., Burbrink, F. T., and J. J. Wiens. 2013. A phylogeny and updated classification of 598 Squamata, including 4161 species of lizards and snakes. BMC Evol Biol. 13:93.

599 R Core Team. 2015. R: A language and environment for statistical computing. R Foundation for 600 Statistical Computing, Vienna, Austria. URL http://www.R-project.org/.

601 Rabosky, D. L., and E. E. Goldberg. 2015. Model inadequacy and mistaken inferences of trait602 dependent speciation. Syst Biol. 64:340-355.

603 Roze, J. A. 1996. Coral Snakes of the Americas: Biology, Identification, and Venoms. Krieger 604 Publishing Company, Malabar, FL.

605 Santos, J. C., L. A. Coloma, and D. C. Canatella. 2003. Multiple, recurring origins of aposematism and diet specialization in poison frogs. Proc Natl Acad Sci USA. 100:12792-12797. 
607 Savage, J. M. 2002. The amphibian and reptiles of Costa Rica: herpetofauna between two continents, 608 between two seas. Chicago Univ. Press, Chicago, IL.

609 Savage, J. M., and J. B. Slowinski. 1992. The colouration of the venomous coral snakes (family

610 Elapidae) and their mimics (families Aniliidae and Colubridae). Biol J Linn Soc. 45:235-254.

611 Sawaya, R. J., O. A. V. Marques, and M. Martins. 2008. Composição e história natural das serpentes de 612 Cerrado de Itirapina, São Paulo, sudeste do Brasil. Biota Neotrop. 8(2):0-0. doi:10.1590/S1676$613 \quad 06032008000200015$.

614 Sazima, I, and A. S. Abe.1991. Habits of five Brazilian snakes with coral-snake pattern, including a 615 summary of defensive tactics. Stud Neotrop Fauna Environ. 26(3):159-164.

616 Smith, S. M. 1975. Innate recognition of coral snake pattern by a possible avian predator. Science 187:759-760.

618 Smith, S. A., and C. Dunn. 2008. Phyutility: a phyloinformatics utility for trees, alignments, and 619 molecular data. Bioinformatics 24:715-716.

620 Speed, M. P., and G. D. Ruxton. 2005. Aposematism: what should our starting point be? Proc R Soc $621 \quad$ Lond B Biol Sci. 272:431-438.

622 Speed, M. P., Brockhurst, M. A., and Ruxton G. D. 2010. The dual benefits of aposematism: Predator 623 avoidance and enhanced resource collection. Evolution. 64(6):1622-1633.

624 Stadler, T. 2009. On incomplete sampling under birth-death models and connections to the sampling625 based coalescent. J Theor Biol. 261:58-66.

626 Stevens, M., and S. Merilaita. 2009. Animal camouflage: current issues and new perspectives. Philos 627 Trans R Soc Lond B Biol Sci. 364:423-427.

628 Stevens, M., and Ruxton, G. D. 2012. Linking the evolution and form of warning coloration in nature. $629 \quad$ Proc R Soc Lond B Biol Sci. 279:417-426.

630 Tanaka, K. 2005. Thermal aspects of melanistic and stripped morphs of the snake Elaphe 
632 Thayer, G.H. 1918. Concealing-coloration in the animal kingdom, ed. 2. New York.

633 Titcomb, G. C., D. W. Kikuchi, and D. W. Pfennig. 2014. More than mimicry? Evaluating scope for 634 flicker-fusion as a defensive strategy in coral snake mimics. Curr Zool. 60:123-130.

635 Tozetti, A. M., R. B. Oliveira, and G. M. F. Pontes. 2009. Defensive repertoire of Xenodon dorbignyi 636 (Serpentes, Dipsadidae). Biota Neotrop. 9:157-163.

637 Uetz, P., and J. Hosek (eds.). 2014. The Reptile Database, http://www.reptile-database.org/.

638 Wallace, A. R. 1867. Mimicry and other protective resemblances among animals. Westminster Foreign 639 Q. Rev. 32:1-43.

640 Wilson, D., R. Heinsohn, and J. A. Endler. 2007. The adaptive significance of ontogenetic colour 641 change in a tropical python. Biol Lett. 3:40-43.

642 Yule, G. U. 1924. A mathematical theory of evolution, based on the conclusions of Dr J. C. Willis.

643 Philos Trans R Soc Lond B Biol Sci. 213:21-87.

644 Zaher, H., F. G. Grazziotin, J. E. Cadle, R. W. Murphy, J. C. Moura-Leite, and S. L. Bonatto. 2009.

645 Molecular phylogeny of advanced snakes (Serpentes, Caenophidia) with an emphasis on South

646 American Xenodontines: A revised classification and descriptions of new taxa. Pap Avul de Zool

$647 \quad 49: 115-153$.

648 Zweifel, R. G. 1960. Herpetology of the Tres Maria Islands. Results of the Puritan-American Museum 649 of Natural History expedition to Western Mexico. Bull Am Mus Nat Hist. 119:77-128.

650 Zwick1, D. J. 2011. GARLI 2.0 https://www.nescent.org/wg_garli/main page. 


\section{FIGURE LEGENDS}

653

654 Figure 1. Maximum clade credibility (MCC) tree of the family Dipsadidae showing the number of 655 species assigned to each color category (center) and the Maximum Likelihood (ML) ancestral state 656 estimate for the color categories: coral-mimic versus non-mimic (left) and contrasting versus cryptic

657 (right). Each color of the stacked bar chart (center) correspond to characteristics of the color patterns; 658 species that do not show contrasting color patterns (i.e., cryptic coloration) are shown in gray, species 659 with contrasting color patterns but that are not supposed mimics of coral snakes are shown in yellow, 660 and species that show contrasting color patterns and are considered mimics of coral snakes are shown 661 in red. The legend in the center of the plate applies different combinations of gray, yellow, and red to 662 show the number of species assigned to the color patterns: coral-mimic (red) versus non-mimic (gray 663 and yellow) and contrasting (yellow and red) versus cryptic (gray). The phylogeny on the left shows 664 the ML ancestral estimate for coral-mimic in red and non-mimic in gray. The phylogeny on the right 665 shows the ML ancestral estimate for contrasting in yellow and cryptic in gray. Both reconstructions 666 were made under the trait-independent BiSSE model. The colored genera correspond to those 667 associated with a tendency for higher speciation rates as a result of the MEDUSA analyses (compare 668 with Figure S3). The tribe Alsophiini is highlighted in green, the two genera belonging to the tribe 669 Hydropsini are marked in blue, and the taxa in red belong to the Tachymenini. Support for the nodes of 670 the MCC tree are provided in Figure S1.

672 Figure 2. Posterior distributions of net diversification rates under the trait-dependent and trait673 independent BiSSE models. Estimates are the joint posterior distribution from $100 \mathrm{MCMC}$ BiSSE runs 674 with randomly sampled trees. Prior distributions for the MCMC searches are shown in blue (unmarked 675 distributions), the horizontal lines below each posterior distribution represent the $95 \%$ confidence 676 interval, and the vertical hashed lines show median values. Top: Trait-dependent diversification rates of 
677

678 model with cryptic color pattern in gray and contrasting pattern in red. Both charts show the single

679 diversification rate of the trait-independent model in yellow. Each posterior distribution is also

680 identified by the letters (a), (b), and (c).

681

682 Figure 3. Posterior distributions of transition rates under the trait-dependent and trait-independent

683 BiSSE models. Estimates are the joint posterior distribution from $100 \mathrm{MCMC}$ BiSSE runs with

684 randomly sampled trees. Prior distributions for the MCMC searches are shown in blue (unmarked

685 distributions), the horizontal lines below each posterior distribution represent the $95 \%$ confidence

686 interval, and the vertical hashed lines show median values. Top: Transition rates from the non-mimic to

687 the coral-mimic (q01 - red) color pattern and back (q10 - gray). Bottom: Transition rates from the

688 cryptic to the contrasting ( $\mathrm{q} 01$ - red) color pattern and back (q10 - gray). Each chart shows results from

689 the trait-dependent and trait-independent BiSSE models identified with different shades of the

690 correspondent transition color and the reference letters (a), (b), (c), and (d).

691

692 Figure 4. Results from the posterior predictive simulations for BiSSE under the trait-dependent (top

693 row - red) and the trait-independent model (bottom row - blue). Simulation parameters drawn from the

694 joint posterior distribution of each BiSSE model estimated from the observed data (categories

695 contrasting and cryptic) and phylogenetic trees. At each replicate a phylogeny was simulated under the

696 BiSSE model until the sum of branch lengths from root to tip of the tree was equal to 1. Left column:

697 Total number of species in the resulting phylogenies. Vertical dashed lines show the richness of the

698 Dipsadidae family used in our analysis (594 spp.). Right column: Relative frequency of the state 0

699 (cryptic). Vertical dashed lines show the observed frequency of cryptic species in the data (0.64). Note

700 that both BiSSE models show similar deviations from the observed data. 
bioRxiv preprint doi: https://doi.org/10.1101/042440; this version posted March 5, 2016. The copyright holder for this preprint (which was not certified by peer review) is the author/funder, who has granted bioRxiv a license to display the preprint in perpetuity. It is made available under aCC-BY-ND 4.0 International license.

702 Figure 1.

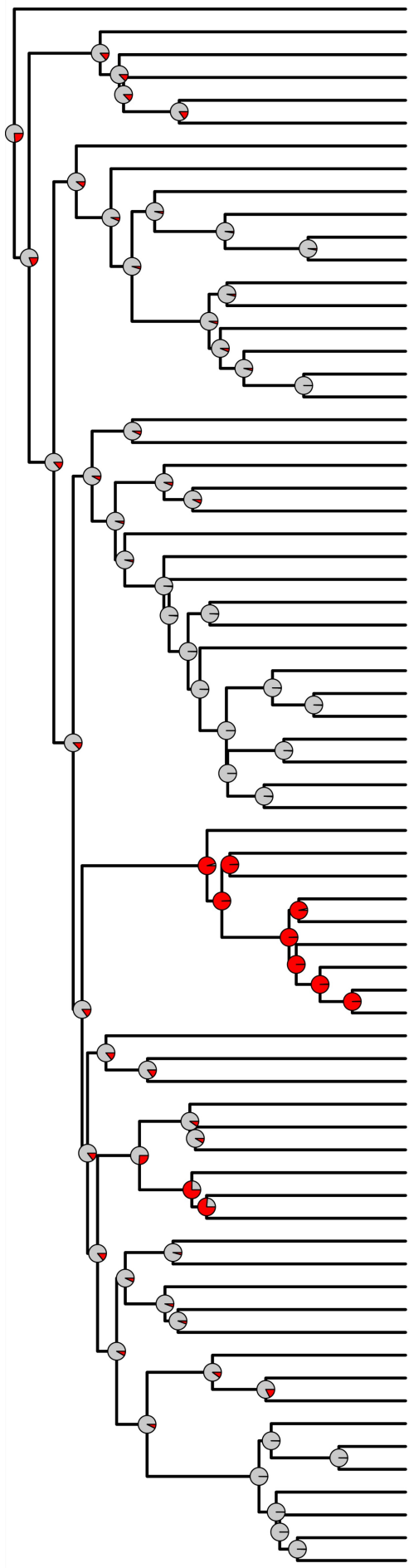

Coral-mimic

$\hookrightarrow$ Non-mimic

Number of species and proportion of states

\begin{tabular}{rl|l|} 
Thermophis & \\
Diadophis & & \\
Heterodon & & \\
Contia & & \\
Farancia & & \\
Carphophis & & \\
Trimetopon \\
Hypsiglena \\
Tretanorhinus \\
Imantodes \\
Leptodeira \\
Nothopsis \\
Geophis \\
Atractus
\end{tabular}

Ninia $\square$

Sibon 11

Dipsas

Sibynomorphus

Psomophis $\square$

Pseudalsophis $\square$

Lygophis $\square$

Xenodon

Eythrolamprus

Uromacer $\square$

Arrhyton $\square$

Haitiophis

Magliophis

Alsophis $\square$

Borikenophis $\square$

Schwartzophis $\square$

Hypsirhynchus

Antillophis ?

Cubophis $\square$

Caraiba

Darlingtonia

laltris 1

Siphlophis

Oxyrhopus

Phimophis $\square$

Mussurana

Rhachidelus

Drepanoides i

Pseudoboa

Boiruna

Clelia 피

Xenopholis $\square$

Hydrodynastes $\square$

Caaeteboia

Sordellina :

Taeniophallus

Echinanthera $\square$

Phalotris

Elapomorphus

Apostolepis $\square \square$

Tropidodryas

Philodryas

Manolepis

Crisantophis

Conophis $\square$

Helicops $\square$

Hydrops in

Pseudoeryx

Calamodontophis

Pseudotomodon

Tachymenis $\square$

Tomodon

Thamnodynastes

Gomesophis

Ptychophis

$\square$ Coral-mimic

$\square$ Non-mimic

Contrasting

Cryptic
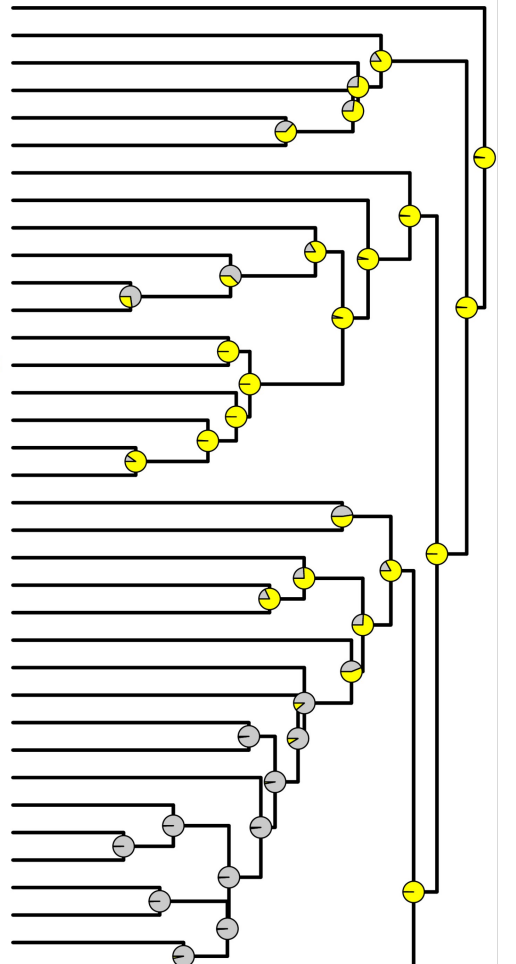

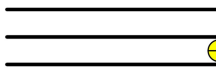

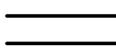
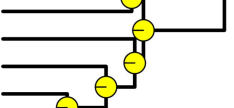

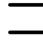

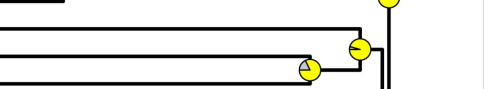

$\longrightarrow$

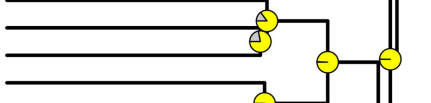

$\longrightarrow$

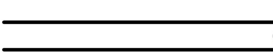

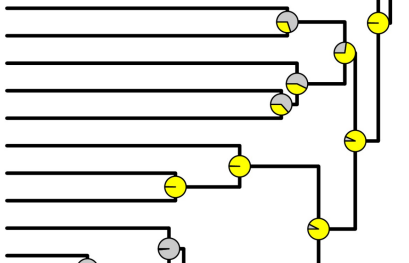

$\theta$

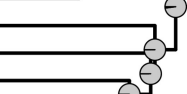

$\supset$ Contrasting

$\supset$ Cryptic 


\section{Figure 2.}

705
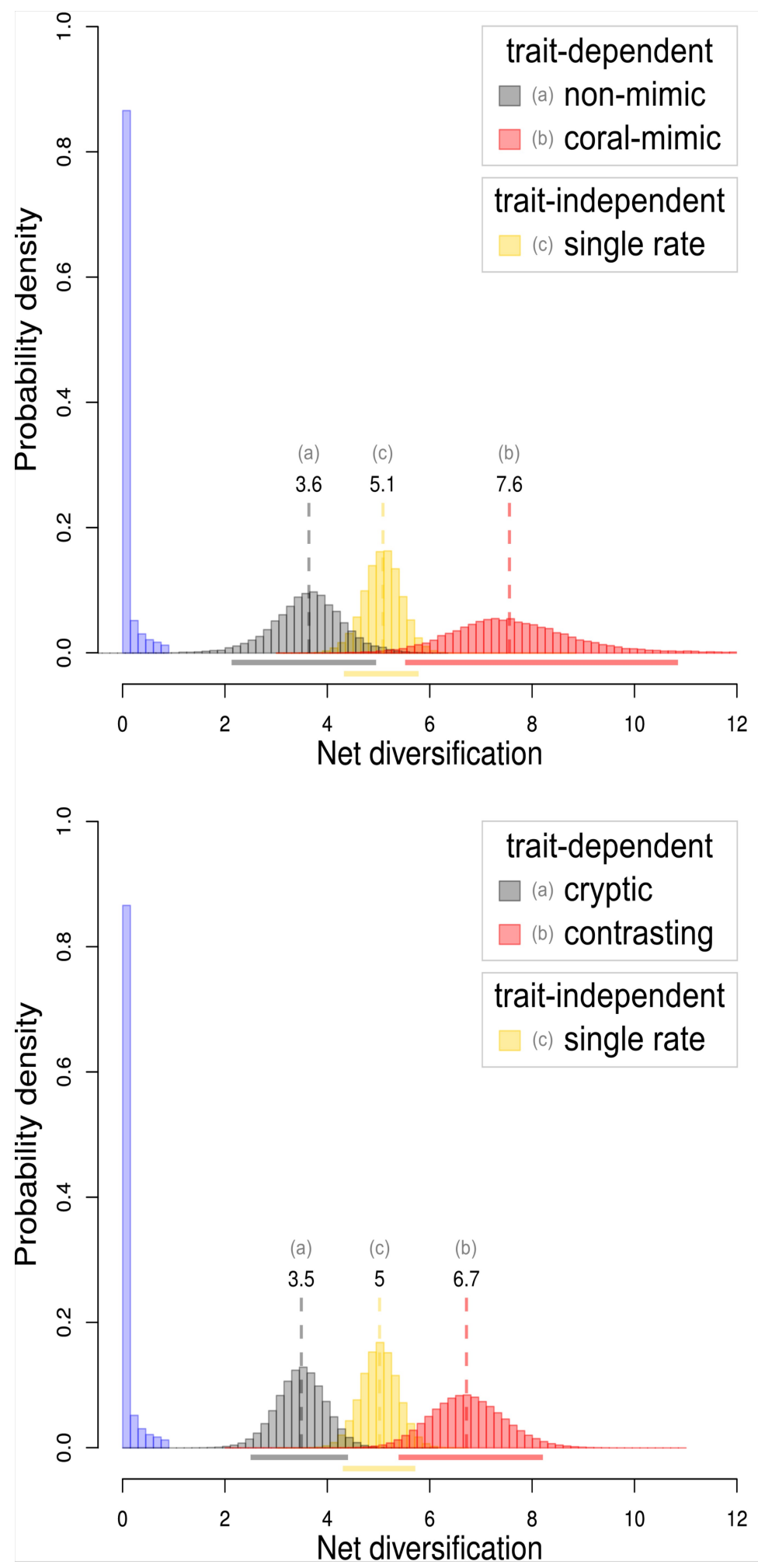


\section{Figure 3.}

707

708
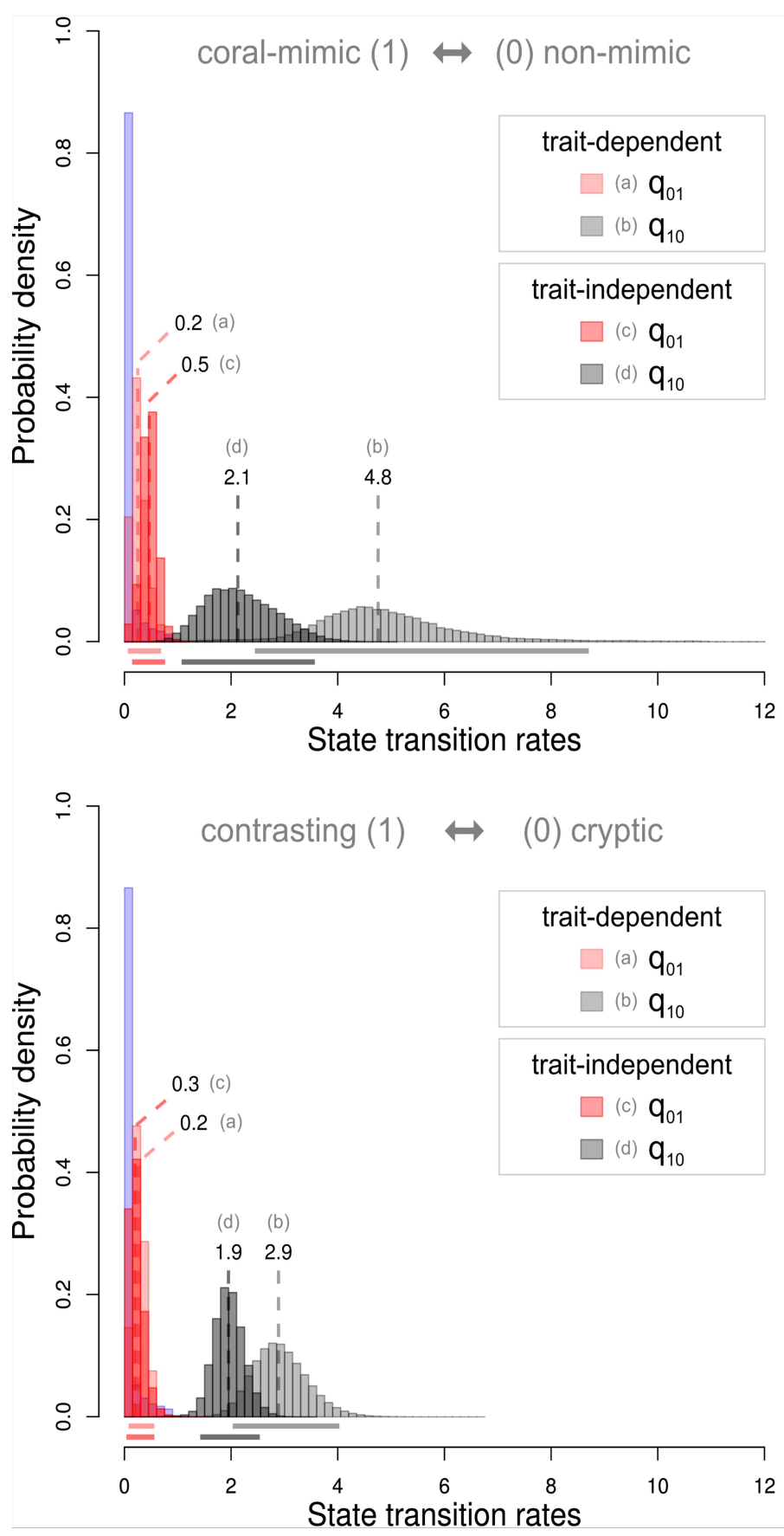


\section{Figure 4.}
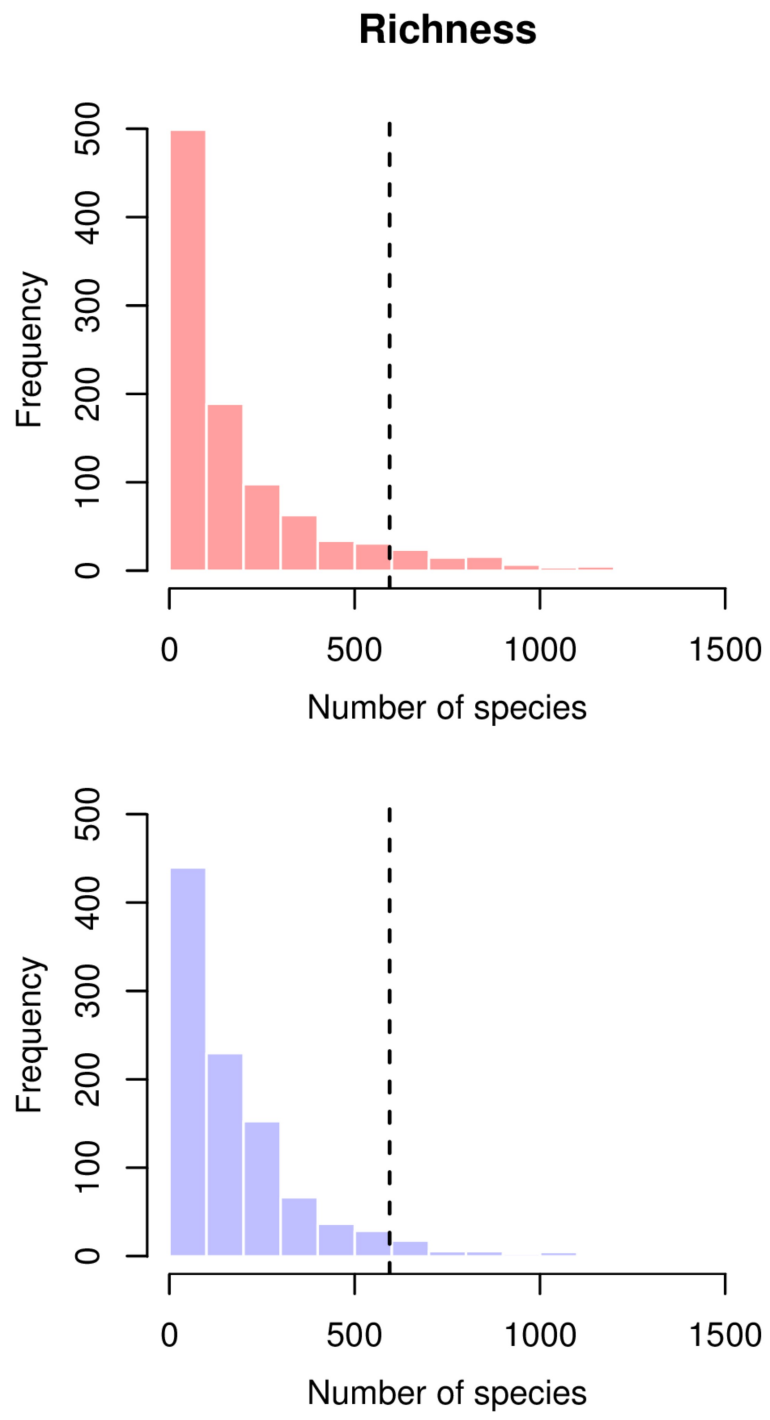

\section{State frequency}
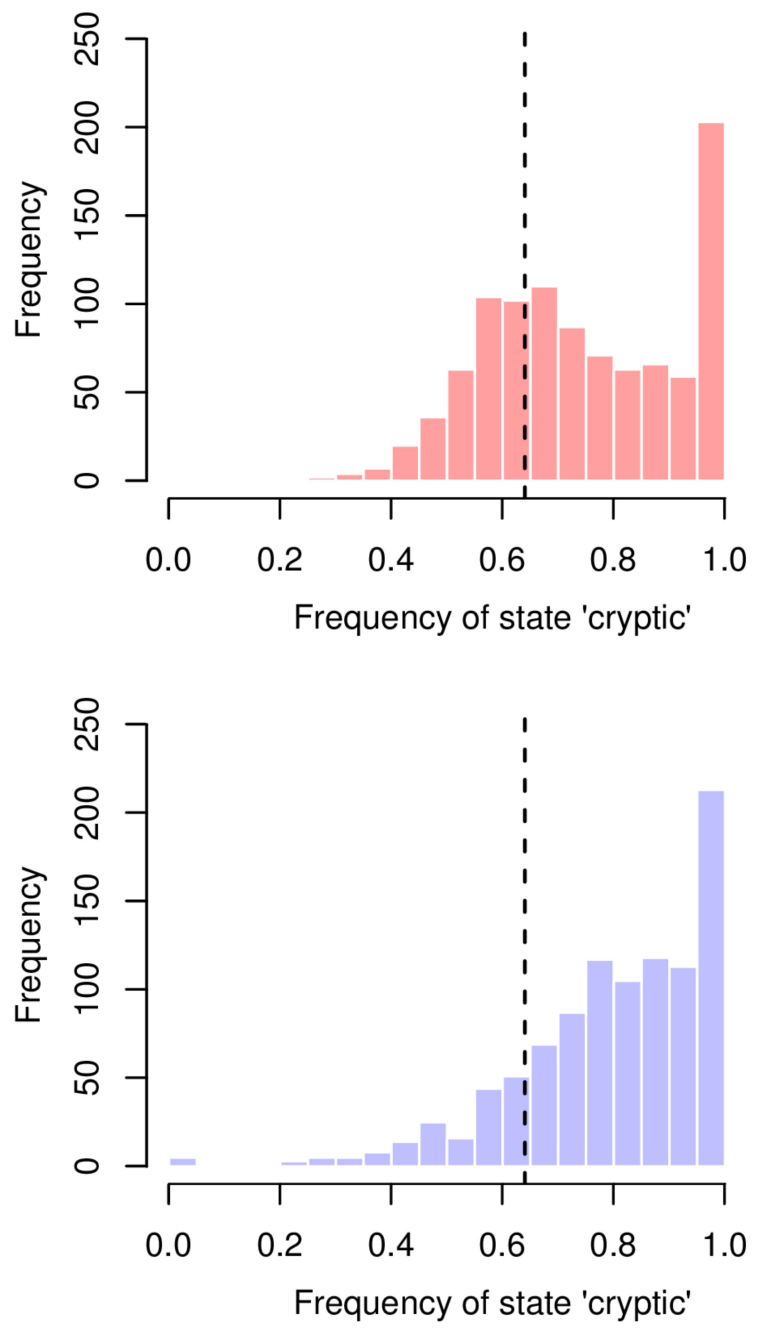
Figure S1: Maximum clade credibility tree with all species used in the analysis. Values in the branches are the respective posterior probabilities for each node. Species are named with the two first letters of the genus followed by the complete species name. Refer to the Table 1 in FigShare (http://dx.doi.org/10.6084/m9.figshare.831493) for the complete name of each species. See Material and Methods of the main text for more information on the methods of tree inference.

Figure S2: Results of model selection using the Bayesian Deviance Information Criteria (DIC) for the coral-mimic versus non-mimic (left) and contrasting versus cryptic (right). DIC values calculated across 100 randomly sampled trees. Values are DIC scores for the trait-dependent model (full model) subtracted from the scores for the trait-independent model (constrained model). Large values - larger than 4 units as a rule of thumb - are expected if the trait-dependent model is to be preferred over the simpler trait-independent model.

Figure S3: Results of the MEDUSA analyses across 100 trees sampled from the posterior distribution of trees generated by the BEAST analysis. The box plots show the distribution of rates of speciation estimated under a Yule model. The colors green, blue, and red denote genera that show a tendency for higher rates of speciation when compared to the background rates shown in gray. The order of genera and color coding is the same used in the Figure 1. Genera shown in green belong to the tribe Alsophiini, in blue to the tribe Hydropsini, and in red to the tribe Tachymenini. 


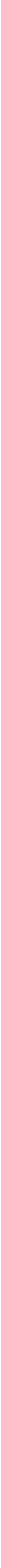


coral-mimic vs. non-mimic

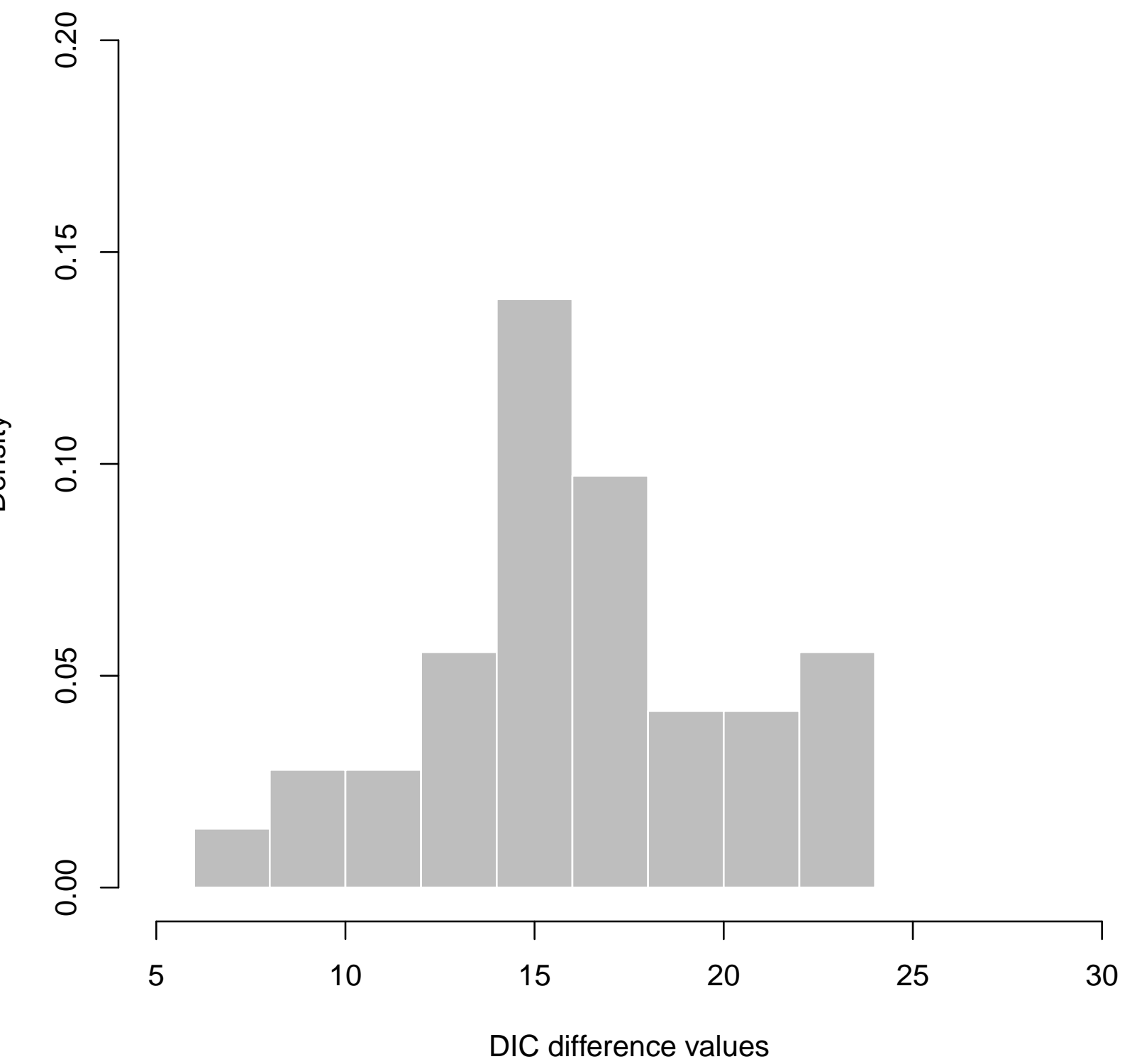

contrasting vs. cryptic

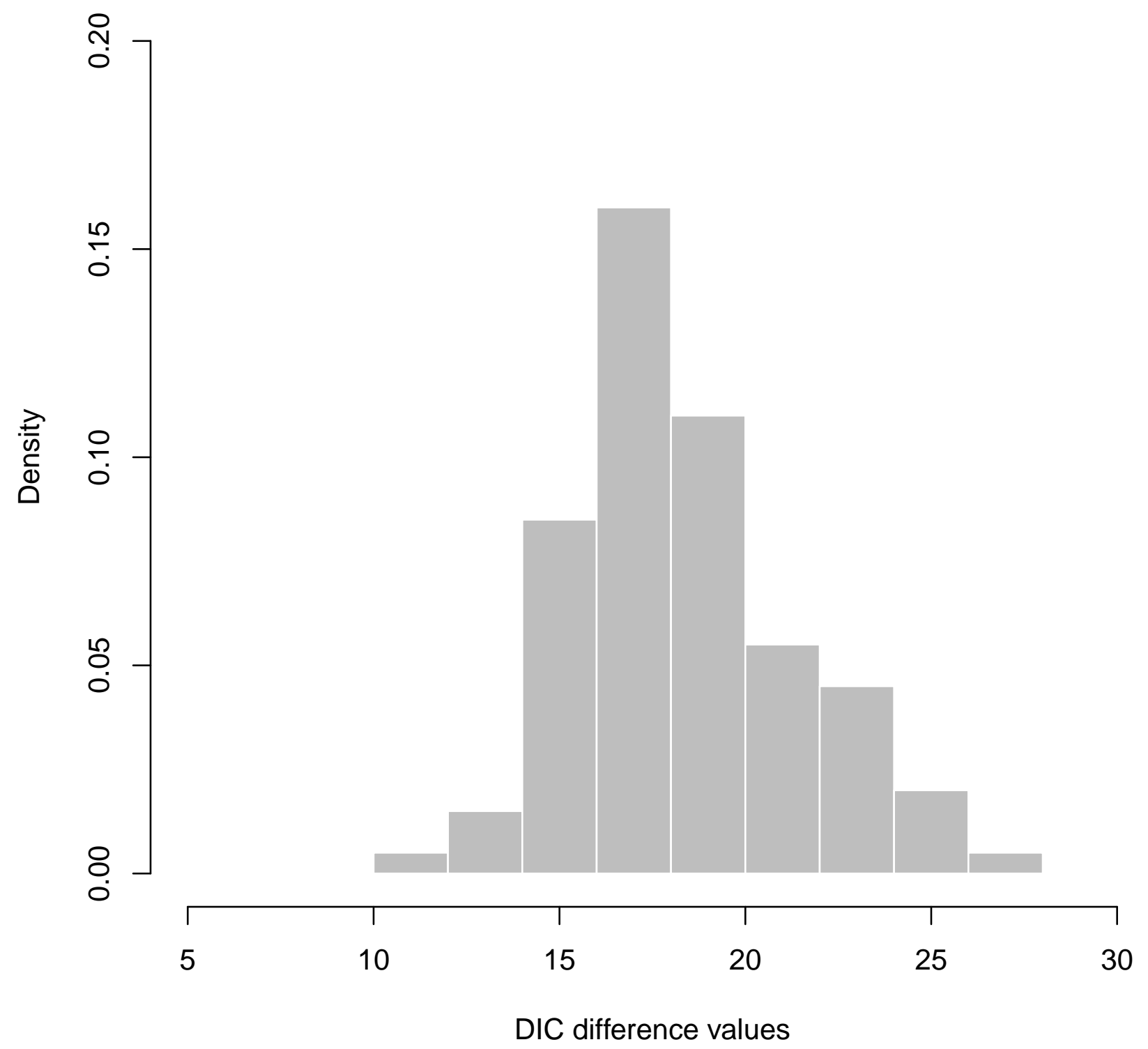




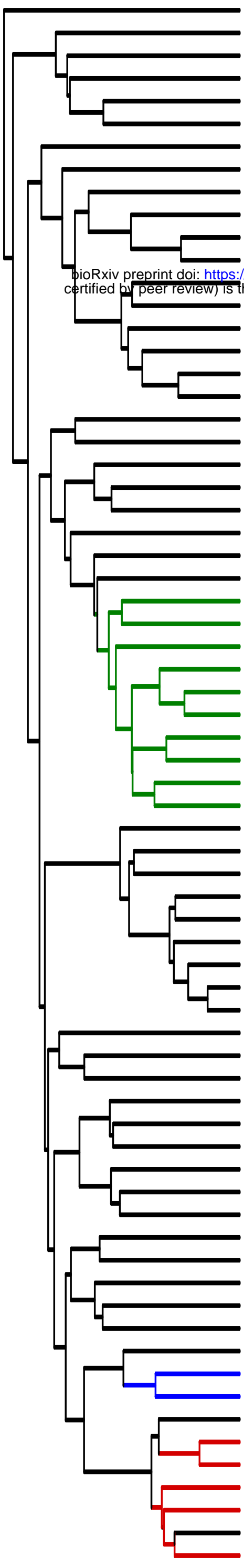

Thermophis

Diadophis

Heterodon

Contia

Farancia

Carphophis

Trimetopon

Hypsiglena

Tretanorhinus

Imantodes

Leptodeira

Nothopsis

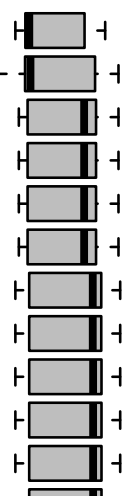

$\begin{array}{ll}\bullet & \\ - & \bullet \\ - & \bullet\end{array}$

$+\rightarrow-1$

hioRxiv preprint doi: https://doi.org/10.1101/042440; this ve posted March 5, 2016. The copyright holder for this preprint (which was not Atractus aCC-BYT-NDT International dicense.

Ninia

Sibon

Dipsas

Sibynomorphus

Psomophis

Pseudalsophis

Lygophis

Xenodon

Erythrolamprus

Uromacer

Arrhyton

Haitiophis

Magliophis

Alsophis

Borikenophis

Schwartzophis

Hypsirhynchus

Antillophis

Cubophis

Caraiba

Darlingtonia

laltris

Siphlophis

Oxyrhopus

Phimophis

Mussurana

Rhachidelus

Drepanoides

Pseudoboa

Boiruna

Clelia

Xenopholis

Hydrodynastes

Caaeteboia

Sordellina

Taeniophallus

Echinanthera

Phalotris

Elapomorphus

Apostolepis

Tropidodryas

Philodryas

Manolepis

Crisantophis

Conophis

Helicops

Hydrops

Pseudoeryx

Calamodontophis

Pseudotomodon

Tachymenis

Tomodon

Thamnodynastes

Gomesophis

Ptychophis
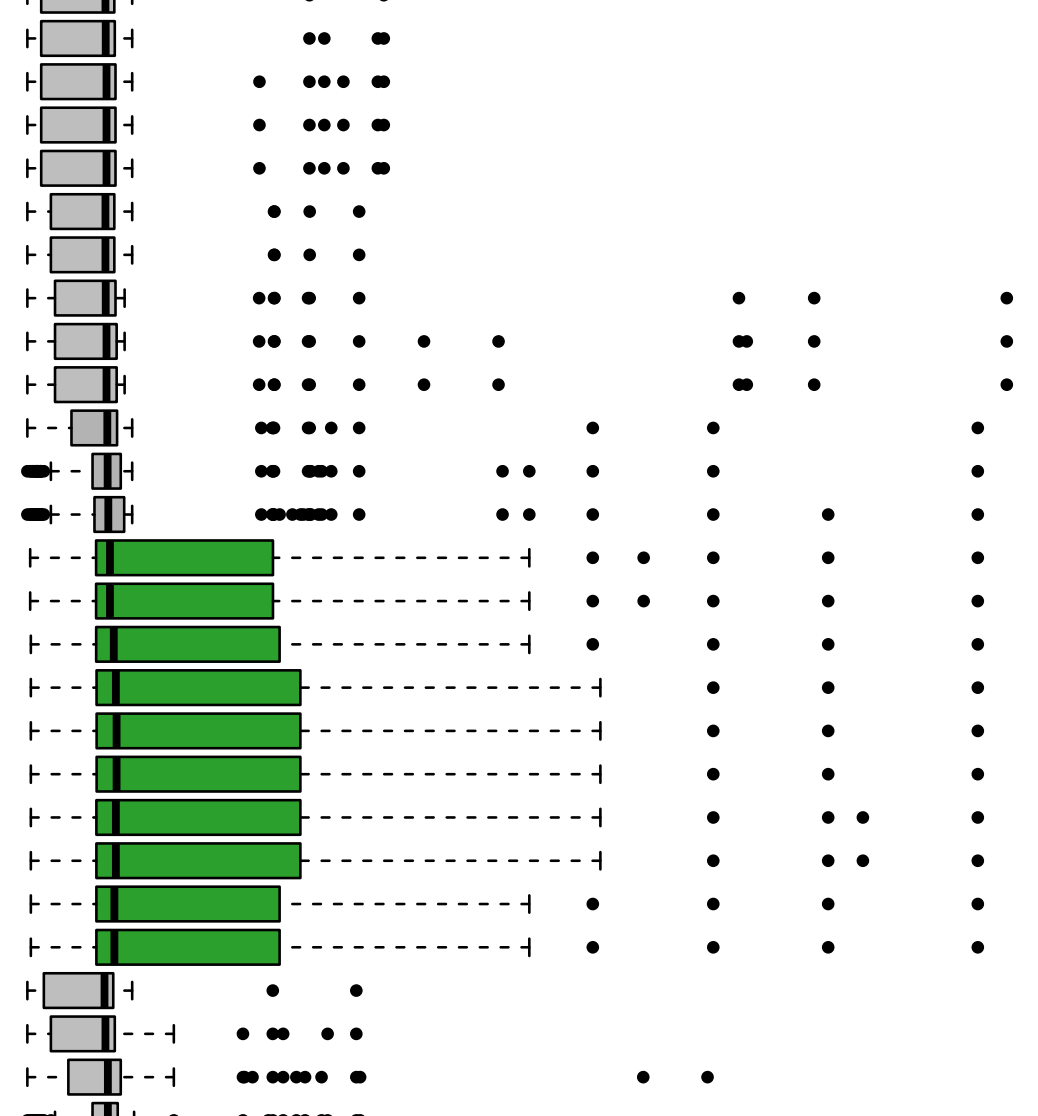

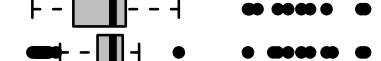

a - - -

$r-\square--1 \quad \bullet \infty \cdots$

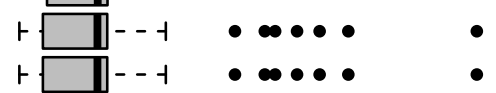

$r-\square--1 \quad \infty \infty \infty$

+-1
$+\square-1$
$+\square-1$

+-1
$+\square-1$

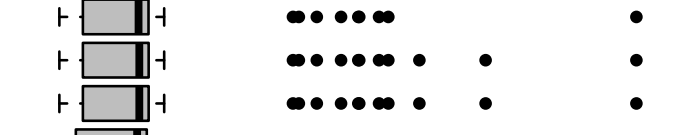

$\vdash \square-1 \quad \cdots \cdots \cdots$

ए

ᄂ

$\vdash \square-1 \quad \bullet \bullet \bullet \bullet$

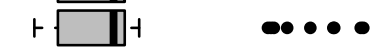

$+-\square$

$\infty \bullet \bullet$

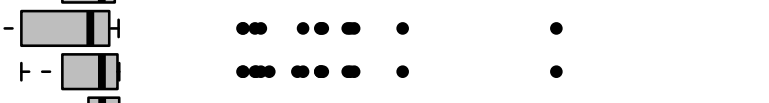

$\bullet \vdash-1-1 \quad \bullet 000 \bullet \bullet \quad \bullet \bullet \bullet \bullet$

1--

r- - प

ト- -

1.- - $\square\left[\begin{array}{l}\square \\ \hline\end{array}\right.$

ト--

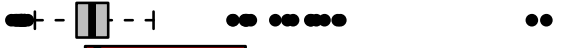

1.-- 